\title{
Estimation of Aerodynamic Parameters in Conditions of Measurement
}

\author{
Moung Htang $\mathrm{Om}^{1 *}$, Kyaw Zin Latt ${ }^{1}$, and Tigran S. Karapetyan ${ }^{1}$ \\ ${ }^{1}$ Russia Moscow Aviation Institute (National Research University), 125080 Moscow, Russia
}

\begin{abstract}
The paper discusses the problem of aircraft parameter identification in conditions of measurement noises. It is assumed that all the signals involved into the process of identification are subjects to measurement noises, that is measurement random errors normally distributed. The results of simulation are presented which show the relation between the noises standard deviations and the accuracy of identification.
\end{abstract}

\section{Introduction}

Identification of aerodynamic parameters could [1-3] be effective for estimating the technical state of the aircraft and the on-board systems $[4,5]$. In this paper the aircraft motion model is formulated which includes the flight parameter sensors and pilot control inputs. For simulation of the measurement noises, the normally distributed discrete random variables with different standard deviations are used.

\section{Simulation of the motion of the aircraft}

The input signal $\left(\varphi_{t}\right)$, generated by the pilot action, is shown in Fig. 1.

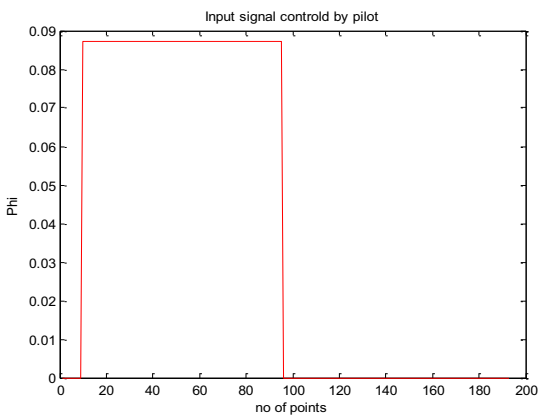

Fig.1. The input signal formed by the action of the pilot.

For simulation, it is necessary to form the object model and to know the output signals. For the formation of object model, the equations for angle of attack and angular velocity of pitch of the aircraft are used. The mathematical formulas in discrete form for the angle of attack (1) and angular velocity of pitch (2) are written as follow

$\alpha\left(t_{i+1}\right)=\alpha\left(t_{i}\right)+\Delta t\left[-Y^{\alpha} \alpha\left(t_{i}\right)+\omega_{z}\left(t_{i}\right)-\right.$ $\left.-Y^{\varphi} \varphi\left(t_{i}\right)\right]$
$\omega_{z}\left(t_{i+1}\right)=\omega_{z}\left(t_{i}\right)+\Delta t\left[M_{z}^{\alpha} \alpha\left(t_{i}\right)+M_{z}^{\omega_{z}} \omega_{z}\left(t_{i}\right)-\right.$ $\left.-M_{z}^{\varphi} \varphi\left(t_{i}\right)\right]$.

Where, $\alpha\left(t_{i+1}\right)$ - angle of attack for time instant $\left(t_{(i+1)}\right)$ (radian); $\omega_{z}\left(t_{i+1}\right)$ - angular velocity of pitch for time instant $\left(t_{(i+1)}\right) \quad($ radian $/ \mathrm{s}) ; \alpha\left(t_{i}\right)$ - angle of attack for time instant $\left(t_{i}\right) ; \omega_{z}\left(t_{i}\right)$ - angular velocity of pitch for time $\operatorname{instant}\left(t_{i}\right) ; \Delta t=t_{i+1}-t_{i}-$ time discretization interval, $Y^{\alpha}, Y^{\varphi}, \quad M_{z}^{\alpha}, \quad M_{z}^{\omega_{z}}, M_{z}^{\varphi}-$ aerodynamic parameters to be identified.

For simulation it was assumed that the registration frequency ( $f$-registration) is $32 \mathrm{~Hz}$. Therefore, the time sampling interval was $\Delta t=1 / 32 \mathrm{~s}$. As a first step the input signal $\left(\varphi_{t}\right)$, angle of attack (1), and the angular velocity of pitch (2) are simulated without any measurement noises. And the simulated signals without measurement noises are shown in Fig. 2.

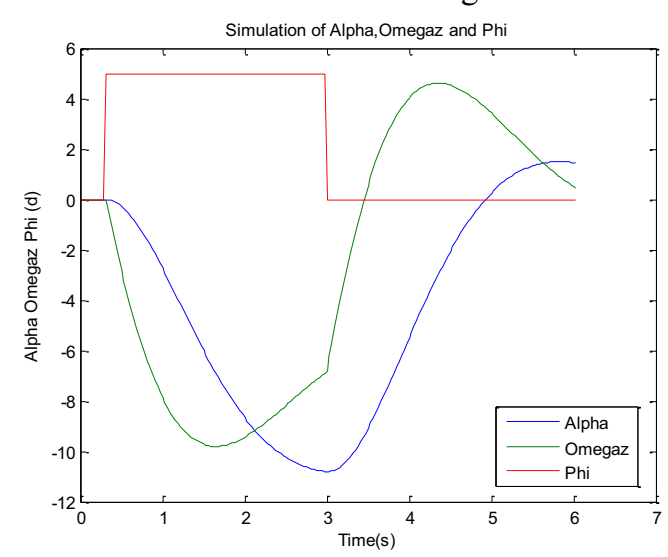

Fig.2. Simulation of the signals without any noises,

After simulation, the measurement of the input signal, angle attack (1) and angular velocity of pitch (2) with measurement noises is performed. The input signal, angle of attack and angular velocity of pitch after being measured with noises are shown in Fig 3. 


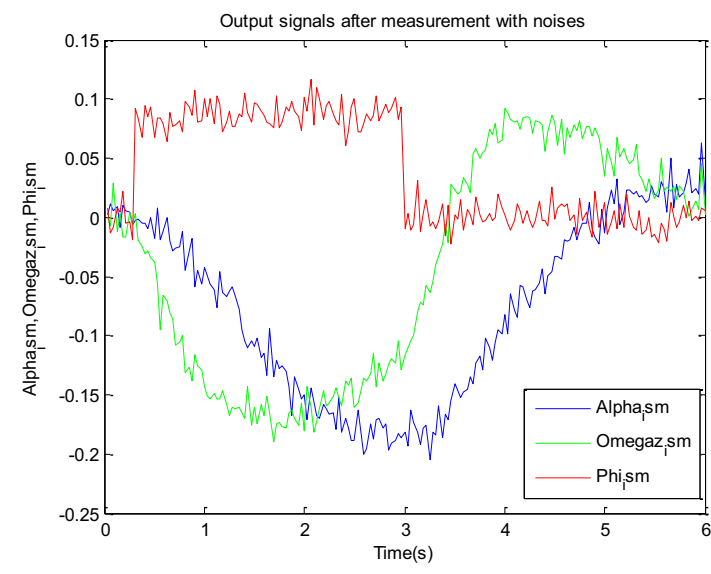

Fig.3. The input signal $\left(\varphi_{t}\right)$, angle of attack and angular velocity after the measurement with noises

\section{Identification of aerodynamic parameters}

For the identification of parameters, it is necessary to form the object model. Let us consider every equation of the object model separately. In general form for an arbitrary instant $t_{i}$ it may be expressed as follows

$$
\begin{gathered}
Y\left(t_{i}\right)=a_{0}+a_{1} x_{1}\left(t_{i}\right)+a_{2} x_{2}\left(t_{i}\right)+a_{3} x_{3}\left(t_{i}\right), \\
i=1,2, \ldots N .
\end{gathered}
$$

Where $a_{0}, a_{1}, a_{2}, a_{3}$ - parameters to be identified;

$$
N \text { - number of samples. }
$$

The object model for identification can be used as a matrix $\mathrm{X}$ and a vector $\mathrm{Y}$ :

$$
\begin{gathered}
\boldsymbol{X}=\left[\begin{array}{cccc}
1 & x_{1 t_{(1)}} & x_{2 t_{(1)}} & x_{3 t_{(1)}} \\
1 & x_{1 t_{(2)}} & x_{2 t_{(2)}} & x_{3 t_{(2)}} \\
1 & x_{1 t_{(N)}} & x_{2 t_{(N)}} & x_{3 t_{(N)}}
\end{array}\right] \\
\boldsymbol{Y}=\left[\begin{array}{c}
Y\left(t_{1}\right) \\
Y\left(t_{2}\right) \\
\ldots \\
Y\left(t_{N}\right)
\end{array}\right] .
\end{gathered}
$$

Where, $X$ - matrix for object model, $Y$ - vector for output signal.

In order to form the matrix $\mathrm{X}$ for the first equation, the input signals $\left(\varphi_{t}\right)$, angle of attack (1), and angular velocity of pitch (2) are used. For the formation of output matrix Y (4) it is better to use the overload, which may be expressed as follow

$$
n_{y}\left(t_{i}\right)=\left[\frac{V}{g}\left(Y^{\alpha} \alpha\left(t_{i}\right)+Y^{\varphi} \varphi\left(t_{i}\right)\right)\right]+R\left(t_{i}\right)
$$

Where, $n_{y}\left(t_{i}\right)$ - overload with specific noise for time instant $\left(t_{i}\right), V-$ airspeed $\left(\mathrm{ms}^{-1}\right), g-$ gravitational acceleration $\left(\mathrm{ms}^{-2}\right), R\left(t_{i}\right)$ - normally distributed random error.

For the purposes of identification it is better to present the second equation in the form

$$
\frac{d \omega\left(t_{i}\right)}{d t}=M_{z}^{\alpha} \alpha\left(t_{i}\right)+M_{z}^{\omega_{z}} \omega_{z}\left(t_{i}\right)-M_{z}^{\varphi} \varphi\left(t_{i}\right)
$$

In this case, the matrix $X$ is the same as in the first equation, and the output vector $\mathrm{Y}$ consists of the estimates for angular velocity time derivative

$$
\frac{d \omega\left(t_{i}\right)}{d t}=\frac{\omega\left(t_{i+1}\right)-\omega\left(t_{i-1}\right)}{2 \Delta t}
$$

After forming all the required matrixes, the least square method (LSM) is used for parameter estimation. This least square method (LSM) is more effective for linear systems. The system which is used in this work is also linear. The least square method is given by

$$
\hat{a}=\left(X^{T} X\right)^{-1} X^{T} Y
$$

Where, $\hat{a}$ - estimates of the parameters; $X$ - matrix for object model; $\mathrm{Y}$ - vector for output signal.

In order to investigate statistically the accuracy of the parameter estimation and the influence of measurement noises, the simulation data and the processing of this data by least square method (LSM) were repeated many times. All the signals involved were distorted with simulated noises, normally distributed. In this work, the experiments were carried out for 6 different levels of the noises standard deviations, 20 independent tests for each level. For each test the LSM estimates of aerodynamic parameters were calculated. Then out of these 20 samples for each aerodynamic parameter the means and standard deviations of the estimates were determined.

It should be explained that a widely known formula [1] associated with the matrix $\left(X^{T} X\right)^{-1}$ for the LSM estimates errors dispersion, is valid in the case of output measurements noises only. In this paper we assume that all the signals used for identification are affected by measurements errors. For this reason we apply the statistical simulation in order to investigate the accuracy of the estimated parameters.

\section{Analysis of the influence of error}

The relative errors for all the estimated parameters are calculated by the following formula

$$
e=\left(\frac{\hat{a}-a_{\text {true }}}{a_{\text {true }}}\right) 100 \%
$$

Where, $\hat{a}$ - the identification estimate of a model parameter; $a_{\text {true }}$ - the true value of the parameter used in the model while generating the test data.

For each test the means and standard deviations of the relative errors were determined. It is noticeable, that the standard deviation of the measurement noise, that is normally distributed random errors, can influence the accuracy of the estimated parameters. By varying the value of standard deviations of these random errors, we could easily notice the different values of the estimated parameters. The relative errors of the estimated parameters. The relative errors of the estimated parameters are shown in Fig. 4, where Sigma is the coefficient proportional to the standard deviation of the measurement noises.

The further analysis of the relative errors is performed for each of estimated parameters in order to know the identification accuracy and to see clearly the confidence boundaries for each estimated parameter. The confidence interval boundaries for the $95 \%$ confidence probability are calculated as follows:

$$
\begin{aligned}
& \text { Sig }_{m}=M[e]+1.96 \sigma[e] \\
& \text { Sig }_{g}=M[e]-1.96 \sigma[e]
\end{aligned}
$$

Where, $\mathrm{Sig}_{m}, \mathrm{Sig}_{g}-95 \%$ confidence interval boundaries for an estimated parameter; $M[e]-$ mean relative error of an estimated parameter, based on 20 
independent tests for each level of the measurement noise; $\sigma[e]$ - standard deviation of a relative error, based on 20 independent tests for each level of the measurement noise.

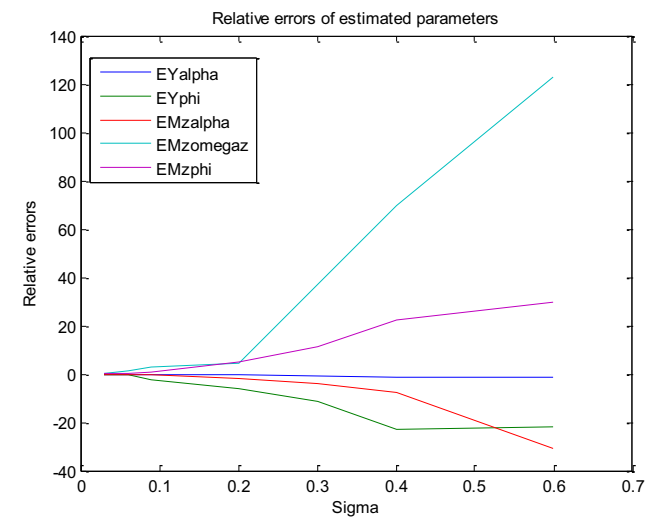

Fig.4. Relative errors of the estimated parameters

The results are presented in Fig. 5-9. The general trend is evident: epy larger is the value of Sigma, that is the level of measurement noises, the broader are the confidence boundaries.

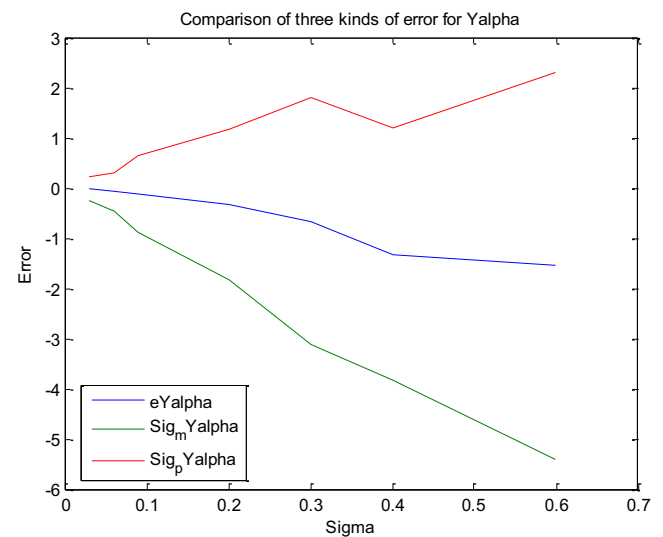

Fig.5. Mean relative error and 95\% confidence interval boundaries for $\left(Y^{\alpha}\right)$.

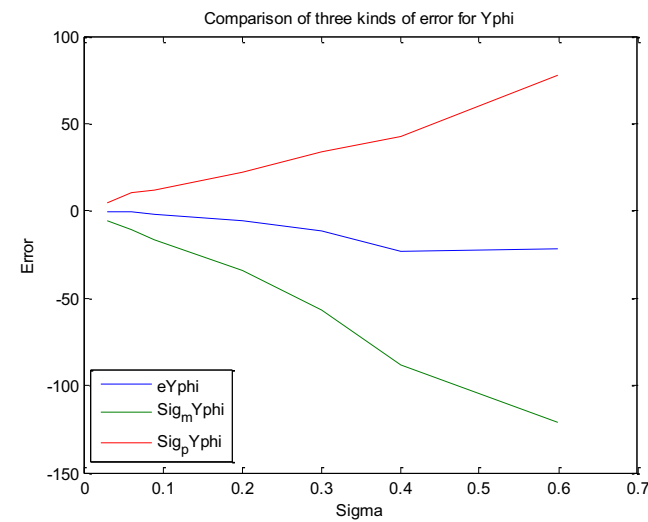

Fig.6. Mean relative error and 95\% confidence interval boundaries for $\left(Y^{\varphi}\right)$.

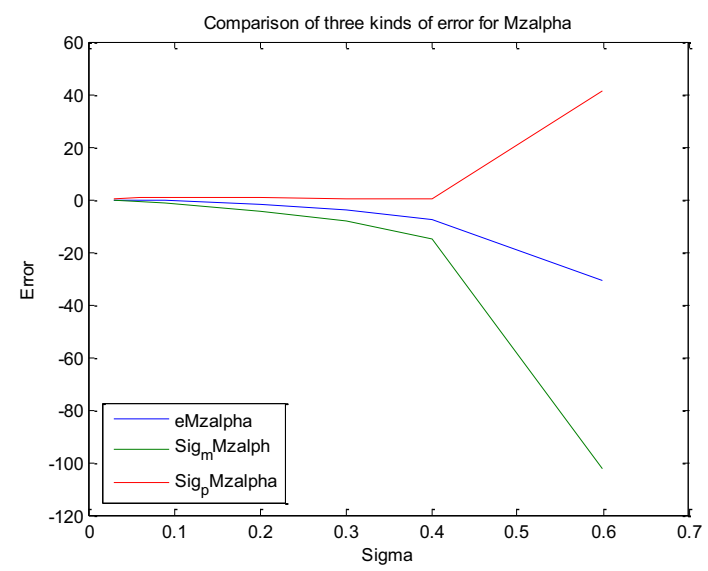

Fig.7. Mean relative error and $95 \%$ confidence interval boundaries for $\left(M_{z}^{\alpha}\right)$.

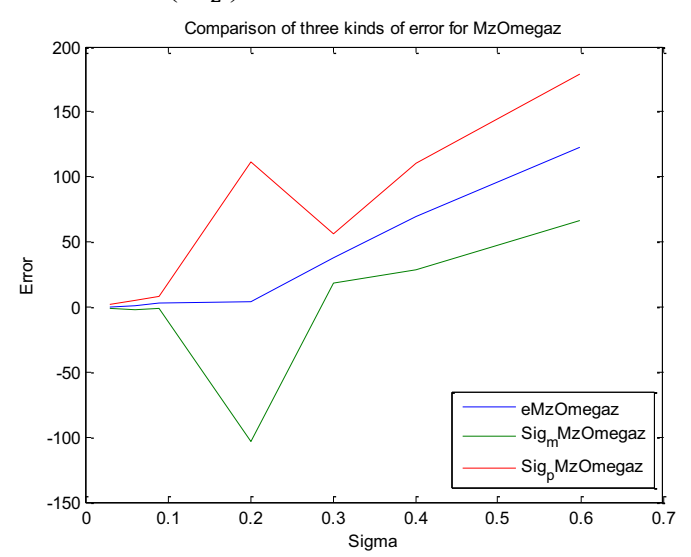

Fig.8. Mean relative error and $95 \%$ confidence interval boundaries for $\left(M_{z}^{\omega_{z}}\right)$.

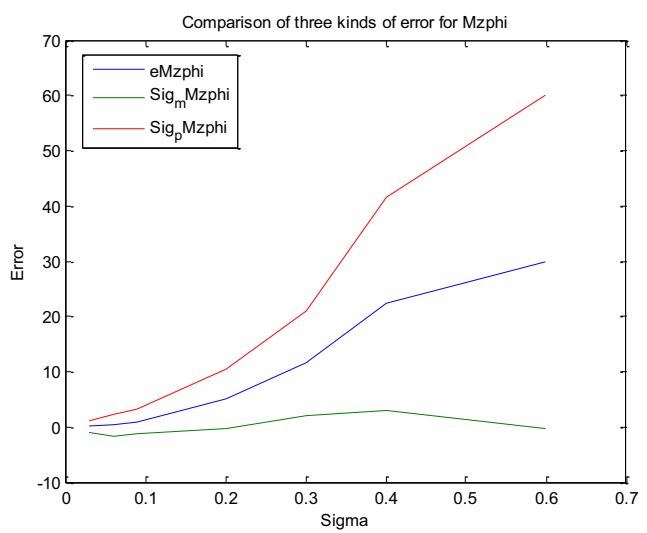

Fig.9. Mean relative error and $95 \%$ confidence interval boundaries for $\left(M_{z}^{\varphi}\right)$.

The above figures present the influence of the average level of noises. In some cases it's better to investigate the influence of noise to signal relation. This relation can be express as

$$
K=\left(\frac{\sigma_{\text {noise }}}{\sigma_{\text {signal }}}\right) \times 100 \%
$$

Where, $\mathrm{K}$ - relation between noise and signal, $\sigma_{\text {noise }}-$ standard deviation of noise, $\sigma_{\text {signal }}$ - standard deviation of signal. 
The results are presented in Fig. 10-14, where in Fig. 10 and Fig. 12 we use noise to signal relation for angle of attack, in Fig. 13 - for angular velocity of pith, and in Fig. 11 and Fig. 14 for input signal $\left(\varphi_{t}\right)$.

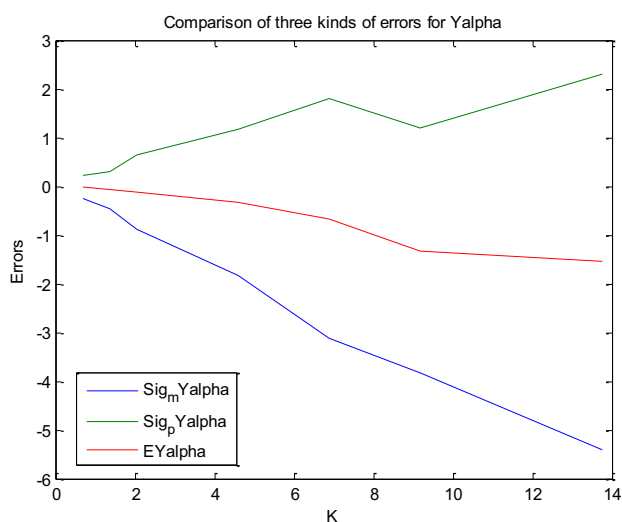

Fig.10. Mean relative error and $95 \%$ confidence interval boundaries depending on the relation between noise and signal $(\mathrm{K})$ for $\left(Y^{\alpha}\right)$.

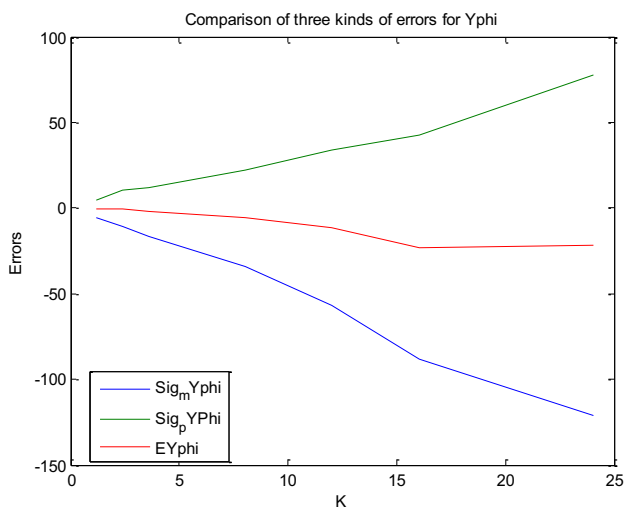

Fig.11. Mean relative error and $95 \%$ confidence interval boundaries depending on the relation between noise and signal $(\mathrm{K})$ for $\left(Y^{\varphi}\right)$.

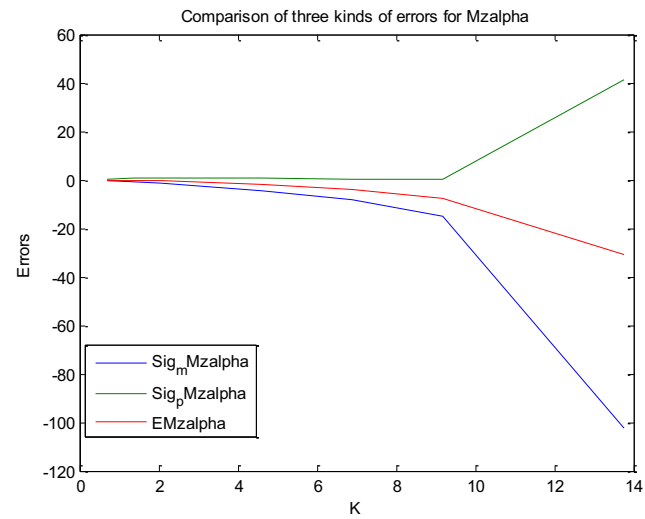

Fig.12 Mean relative error and 95\% confidence interval boundaries depending on the relation between noise and signal $(\mathrm{K})$ for $\left(M_{Z}^{\alpha}\right)$.

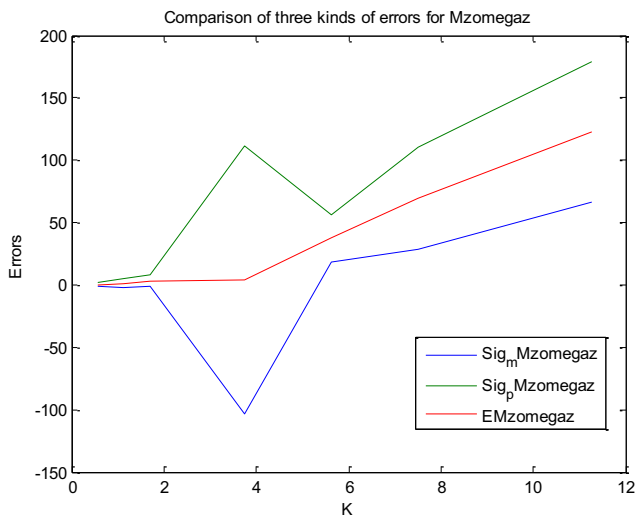

Fig.13. Mean relative error and $95 \%$ confidence interval boundaries depending on the relation between noise and $\operatorname{signal}(\mathrm{K})$ for $\left(M_{Z}^{\omega_{z}}\right)$.

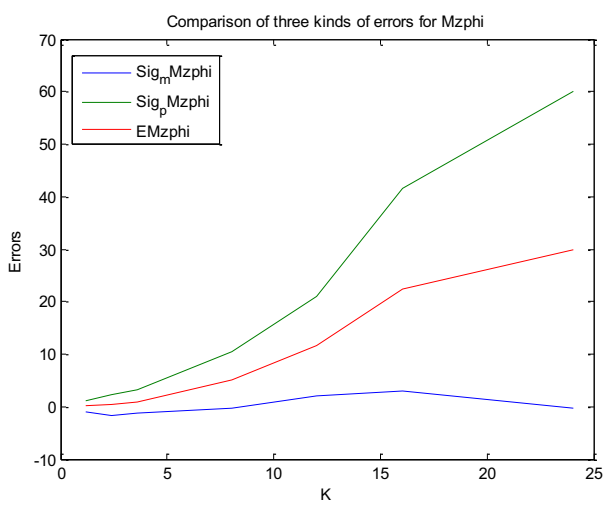

Fig.14. Mean relative error and $95 \%$ confidence interval boundaries depending on the relation between noise and signal $(\mathrm{K})$ for $\left(M_{z}^{\varphi}\right)$.

\section{Conclusion}

The obtained experimental results show that the proposed method of identification for estimating aerodynamic parameters can assure the high accuracy of estimation at considerable levels of noise to signal relation.

\section{References}

1. R.V. Jategaonkar, Flight vehicle system identification: a time domain methodology (American Institute of Aeronautics and Astronautics, Reston, 2006)

2. V. Klein, E.A. Morelli, Aircraft system identification: theory and practice (American Institute of Aeronautics and Astronautics, Reston, 2006)

3. O.N. Korsun, Automation and remote control, 64, 772-781 (2003)

4. O.N. Korsun, B.K. Poplavskii, Journal of Computer and Systems Sciences International, 50, 130-143 (2011)

5. O.N. Korsun, S.V. Nikolaev, S.G. Pushkov, Journal of Computer and Systems Sciences International, 55, pp. 446-457 (2016) 